\title{
NONLINEAR NATURAL FREQUENCIES OF A TAPERED CANTILEVER BEAM
}

\author{
M. Abdel-Jaber ${ }^{1}$, A.A. Al-Qaisia, ${ }^{2, *}$ and M.S. Abdel-Jaber ${ }^{3}$ \\ ${ }^{I}$ Department of Civil Engineering, Faculty of Engineering, Applied Science University, Amman, Jordan \\ ${ }^{2}$ Department of Mechanical Engineering, Faculty of Engineering and Technology, \\ University of Jordan, Amman, Jordan \\ ${ }^{3}$ Department of Civil Engineering, Faculty of Engineering and Technology, \\ University of Jordan, Amman, Jordan \\ *(Corresponding author: E-mail: alqaisia@ju.edu.jo)
}

Received: 5 November 2007; Revised: 17 January 2008; Accepted: 23 January 2008

\begin{abstract}
The non-linear natural frequencies of the first three modes of a clamped tapered beam are investigated. The mathematical model is derived using the Euler-Lagrange method and the continuous system is discretized using the assumed mode method. The resulted uni-modal nonlinear equation of motion was solved using the harmonic balance (HB) to obtain approximate analytical expressions for the nonlinear natural frequencies. Results were obtained for two types of taper; double taper, i.e. the beam width and thickness are varied linearly along the beam axis and single taper "wedge shaped beams", i.e. the variation is in thickness only. The effects of vibration amplitude and taper ratio on the nonlinear natural frequencies for the first three modes are obtained and presented in non-dimensional form.
\end{abstract}

Keywords: Nonlinear, free vibration, harmonic balance, tapered beam, cantilever beam

\section{INTRODUCTION}

It is known that a lot of engineering structures can be modeled as beam. Some can be modeled as tapered beams, such as piles, fixed-type platforms, tower structures, high buildings and robot arms.

In general, due to various excitation loads "wind and waves", high aspect ratio and flexibility such structures might have large deformations and deflections. The prediction of the dynamic behavior is extremely important during the design process.

The linear vibration theory predicts the natural frequencies to be independent of the amplitude. But in many cases, the deflection in structures may reach large values and consequently, using the linear vibration assumption is not valid. In order to take into consideration the nonlinearities arised due to large deformations, the nonlinear vibration theory must be used to predict with high accuracy the dynamic behavior like; natural frequencies and dynamic responses.

In this paper, the non-linear planar large amplitude free vibration of a tapered cantilever beam is studied for two cases; double tapered beam and single tapered 'wedge shaped beam".

Most of the pertinent literature is directed towards the calculation of linear natural frequencies and mode shapes [1-6] with different end conditions and with attached inertia elements at the free end of the beam. In [7], a simple formulation for the large amplitude free vibrations of tapered beams was presented. The method is based on an iterative numerical scheme to obtain results for tapered beams with rectangular and circular cross sections.

The objective of the present work is to extend the results and analysis obtained in [8] to study the non-linear planar large amplitude free vibrations of a cantilever tapered beam for the cases of a double taper beam and a single taper "wedge shaped beam". The mathematical model is derived 
using the Lagrange method and the resulting continuous equation is discretized using the assumed mode method $[9,10]$. The inextensibility condition [11] is used to relate the axial shortening due to transverse deflection in the formulation of the kinetic energy of the beam and the nonlinear curvature is used in the potential energy expression.

\section{MATHEMATICAL MODEL}

\subsection{System Description and Assumptions}

A schematic drawing of the beam under study is shown in Figure 1. The physical properties, modulus of elasticity $E$ and density $\rho$, of the beam are constants. While the beam thickness and width are varied linearly along the beam axis. The beam is clamped at one end and free at the other, the cross sectional area and moment of inertia at the large (Clamped end) are $A_{b 1}$ and $I_{b l}$, respectively.

The thickness of the beam is assumed to be small compared to the length of the beam, so that the effects of rotary inertia and shear deformation can be ignored. The beam transverse vibration can be considered to be purely planar and the amplitude of vibration may reach large values.

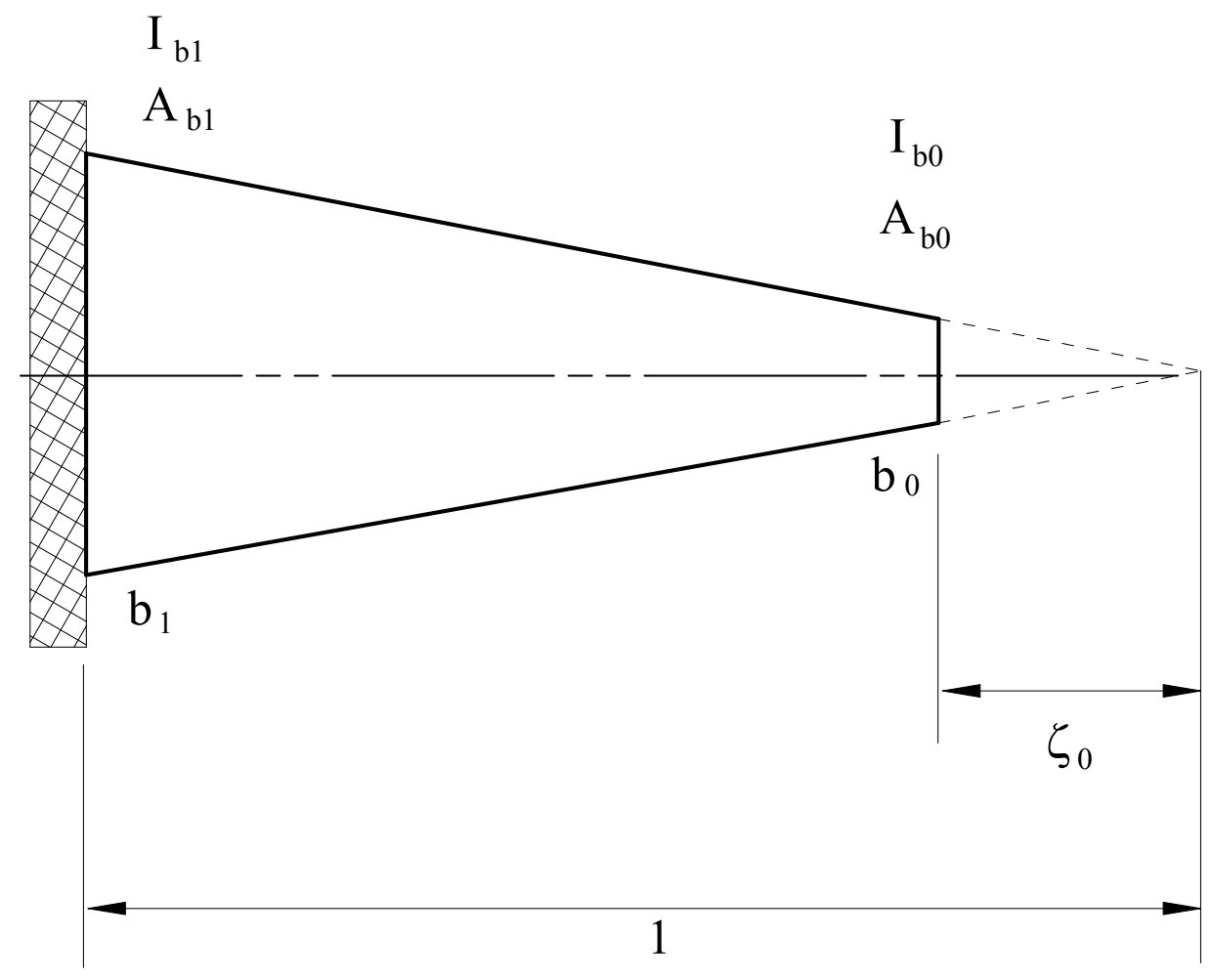

Figure 1. A Schematic Drawing of the Tapered Cantilever Beam 


\subsection{Derivation of the Equation of Motion}

Using the deformed beam, see Figure 2, the potential energy of the beam can be written as

$\left.V=\frac{E l}{2} \int_{0}^{1} I(\zeta) R^{2} d \zeta\right)$

where $\zeta=s / l, I(\zeta)$ is the variable second moment of area and $R$ is the curvature of the beam neutral axis and can expressed as $[8,10]$

$R=\lambda \phi^{\prime}$

where $\lambda=1 / l$, the prime is the derivative with respect to the dimensionless length, $\zeta$, and is the change of the slope along the beam (see Figure 2). In order to express the exact curvature in terms of the transverse deflection, $v$, it is noted that $\cos \phi=\sqrt{1-\sin ^{2} \phi}$. This implies that $\sin \phi=\mathrm{d} v / \mathrm{d} s=\lambda v^{\prime}$ (as in Figure 2). Differentiating $\sin \phi=\lambda v^{\prime}$ with respect to $\zeta$, using the above trigonometric identities, expanding the resulted term in a power series and retaining the terms up to the fourth order, the nonlinear curvature $R$ can be written as

$R^{2}=\lambda^{4}\left(v^{\prime \prime 2}+\lambda^{2} v^{\prime \prime 2} v^{\prime 2}\right)$

The kinetic energy $T$ of the beam can be written as

$T=\frac{1}{2} \rho l \int_{0}^{1} A(\zeta)\left[\dot{u}^{2}+\dot{v}^{2}\right] \mathrm{d} \zeta$

where $u$ is the axial shortening due to bending deformation as can be seen in Figure 2. The inextensibility condition dictates that a total axial shortening $u$ is given by [11]

$\lambda u=\zeta-\int_{0}^{\zeta} \cos \phi d \zeta=\zeta-\int_{0}^{\zeta} \sqrt{1-\left(\lambda v^{\prime}\right)^{2}} d \zeta$

Expanding the radical term in a power series, assuming that $\left(\lambda v^{\prime}\right)^{2}<<1$, the axial shortening can be represented as

$u=\frac{1}{2} \int_{0}^{\zeta}\left(\lambda v^{\prime 2}+\frac{\lambda^{3}}{4} v^{\prime 4}\right) \mathrm{d} \zeta$

Differentiating Eq. 6 with respect to time yields

$\dot{u}=\frac{1}{2} \frac{d}{d t} \int_{0}^{\zeta}\left(\lambda v^{\prime 2}\right) \mathrm{d} \zeta$ 


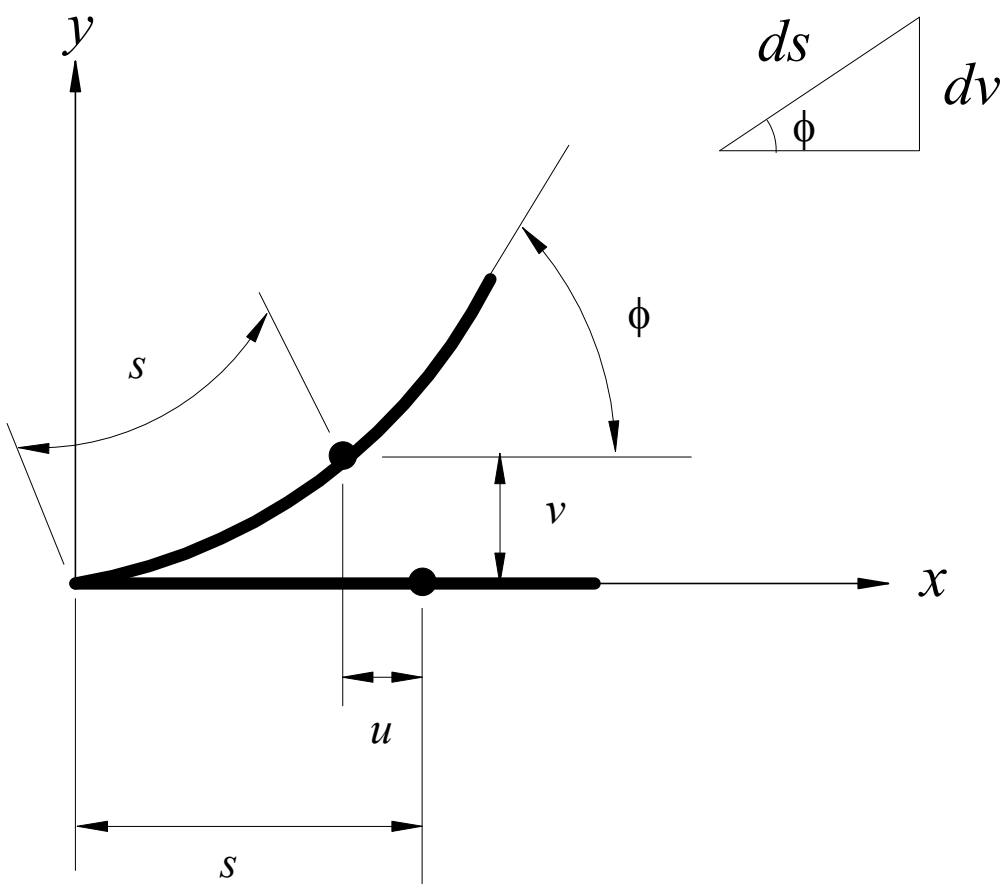

Figure 2. The Deformed Inextensible Beam

The Lagrangian of the beam under consideration can be expressed as

$L=T-V$

It is clear that the continuous system in Eq. 8 does not admit a closed form solution. The interest here is in the case where the beam motion is governed by single active mode. The Lagrangian of the system $L$ can be discretized by using the assumed mode method and substituting

$v(\zeta, t)=\phi_{i}(\zeta) q(t)$

where $\phi_{i}(\zeta)$ is the normalized, self-similar (i.e. independent of the motion amplitude) assumed mode shape of the beam and $q(t)$ is an unknown time modulation of the assumed deflection mode $\phi_{i}(\zeta)$. In the present work $\phi_{i}(\zeta)$ for a double tapered beam is (see reference [6]):

$\phi_{i}(\zeta)=\zeta^{-1}\left[C_{1} J_{2}(Z)+C_{2} Y_{2}(Z)+C_{3} I_{2}(Z)+C_{4} K_{2}(Z)\right]$

where $A(\zeta)=A_{b 1} \zeta^{2}$ and $I(\zeta)=I_{b 1} \zeta^{4}$, and for wedge-type beams (single taper)

$\phi_{i}(\zeta)=\zeta^{-1 / 2}\left[C_{1} J_{1}(Z)+C_{2} Y_{1}(Z)+C_{3} I_{1}(Z)+C_{4} K_{1}(Z)\right]$

And $A(\zeta)=A_{b 1} \zeta$ and $I(\zeta)=I_{b 1} \zeta^{3}$

For both cases $Z=2 \beta \zeta^{1 / 2}, \beta^{4}=\frac{\rho A_{b 1} L_{l}^{4} \omega_{l}^{2}}{E I_{b 1}}, \omega_{l}$ is the linear frequency of vibration, $J$ and $Y$ 
are Bessel functions of the first and second kind, respectively, and $I$ and $K$ are modified Bessel functions of the first and second kind, respectively. $C_{1}, C_{2}, C_{3}$ and $C_{4}$, are arbitrary constants to be determined by imposing the following boundary conditions to both ends of the beam; zero bending moment and zero shear force at the free end and zero deflection and zero slope at the clamped end.

$$
\begin{aligned}
& E I(\zeta) \phi_{i}^{\prime \prime}\left(\zeta_{0}\right)=0 \\
& \frac{\mathrm{d}}{\mathrm{d} \zeta}\left(E I(\zeta) \phi_{i}^{\prime \prime}\left(\zeta_{0}\right)\right)=0 \\
& \phi_{i}^{\prime}(1)=0 \\
& \phi_{i}(1)=0
\end{aligned}
$$

Using Eqs. 7, 9 and 10 or 11 the Lagrangian expression of the tapered beam under consideration can be expressed as, for the $i$-th mode of vibration

$$
L=\rho l^{3}\left(\beta_{1} \dot{q}^{2}+\beta_{2} q^{2} \dot{q}^{2}-\beta^{2} \beta_{3} q^{2}-\beta^{2} \beta_{4} q^{4}\right)
$$

where

$$
\begin{aligned}
& \beta_{1}=\int_{0}^{1} A_{1}^{*} \phi^{2} \mathrm{~d} \zeta \\
& \beta_{2}=\int_{0}^{1} A_{1}^{*}\left\{\int_{0}^{\zeta} \phi^{\prime 2} \mathrm{~d} \chi\right\}^{2} \mathrm{~d} \zeta \\
& \beta_{3}=\int_{0}^{1} I_{1}^{*} \phi^{\prime 2} d \zeta \\
& \beta_{4}=\int_{0}^{1} I_{1}^{*} \phi^{\prime 2} \phi^{\prime \prime 2} \mathrm{~d} \zeta
\end{aligned}
$$

For the double tapered beam; $A_{1}^{*}=A_{b l} \zeta^{2}$ and $I_{1}^{*}=I_{b l} \zeta^{4}$ and for single tapered beam, the wedge beam $A_{1}^{*}=A_{b 1} \zeta$ and $I_{1}^{*}=I_{b 1} \zeta^{3}$.

Applying the Euler-Lagrangian equation to the system Lagrangian

$$
\frac{\mathrm{d}}{\mathrm{d} t}\left(\frac{\partial L}{\partial \dot{q}}\right)-\frac{\partial L}{\partial q}=0
$$

the following non-linear, non-dimensional uni-modal equation of motion is obtained: 
$\beta_{1} \ddot{q}+\beta_{2}\left(q^{2} \ddot{q}+q \dot{q}^{2}\right)+\beta^{2}\left(\beta_{3} q+2 \beta_{4} q^{3}\right)=0$

Due to the fact that, some of the coefficients $\beta_{i}$, defined by Eqs. 14-17, may have large values, Eq. (19) for convenience is scaled to the form;

$\ddot{q}+q+\varepsilon_{1}\left(q^{2} \ddot{q}+q \dot{q}^{2}\right)+\varepsilon_{2} q^{3}=0$

A dot is used to denote a derivative with respect to the non-dimensional time. $t^{*}=\left(\beta^{2} \beta_{3} / \beta_{1}\right)^{1 / 2} t$, $\varepsilon_{1}=\frac{\beta_{2}}{\beta_{1}}$ and $\varepsilon_{2}=\frac{2 \beta_{4}}{\beta_{3}}$ are dimensionless coefficients.

Eq. 20 describes the non-linear non-dimensional planar flexural free vibration of the inextensible tapered beam. In this equation, the terms $\varepsilon_{1} \ddot{q} q^{2}$ and $\varepsilon_{1} q \dot{q}^{2}$ are inertia non-linearities arised from using the inextensibility condition in the kinetic energy and they are of softening type (i.e., they lead to a decrease in the natural frequency when the vibration amplitude increases). The non-linear term $\varepsilon_{2} q^{3}$ is due to the potential energy stored in bending and arises as a result of using non-linear curvature and it is of hardening static type (i.e., it leads to an increase in the natural frequency when the vibration amplitude increases). The nonlinear natural frequencies of the beam are dominated by the two competing non-linearities mentioned above, and the behaviour of the tapered beam considered in this work is either hardening or softening depending on the ratio $\varepsilon_{1} / \varepsilon_{2}[10]$.

\section{METHOD OF SOLUTION}

The calculations of the coefficients $\beta_{i}$ in Eqs. 14-17, $\varepsilon_{1}$ and $\varepsilon_{2}$ indicate that the non-linear oscillator described in Eq. 20 is strongly nonlinear, and the nonlinear natural frequencies are calculated using the Harmonic Balance method (HB). The initial conditions are taken to be $q(0)=A$ and $\dot{q}(0)=0$ where $A$ is the amplitude of the motion.

According to the HB method, an approximate single term solution (SHB) $[8,9]$ takes the form

$q\left(t^{*}\right)=A \cos \left(\omega t^{*}\right)$

where $\omega$ is the non dimensional nonlinear natural frequency, i.e. the ratio of the nonlinear frequency to the linear one. Substituting Eq. 21 and its derivatives into Eq. 20 and equating coefficients, one obtains

$\omega^{2}=\frac{1+(3 / 4) \varepsilon_{2} A^{2}}{1+\left(\varepsilon_{1} A^{2} / 2\right)}$

To improve the accuracy of the assumed solution, more terms can be added and a two term solution is sought (2THB), such that

$q\left(t^{*}\right)=A_{1} \cos \left(\omega t^{*}\right)+A_{3} \cos \left(3 \omega t^{*}\right)$

As one can see, the added term is of order three and this is due to the fact that the nonlinear terms 
in Eq. $20 " \varepsilon_{1} \ddot{q} q^{2}, \varepsilon_{1} q \dot{q}^{2}$ and $\varepsilon_{2} q^{3}$ "are odd and of order three.

Using the above mentioned initial conditions, yields

$A=A_{1}+A_{3}$

Substituting Eq. (23) and their derivatives into Eq. (20) and equating the coefficient of each of the assumed harmonics, one obtains

$$
\begin{aligned}
& A_{3}=\frac{\left(\varepsilon_{2} / 4\right)\left(A_{1}^{3}+3 A_{3}^{3}\right)-\left(\varepsilon_{1} \omega^{2} / 2\right)\left(A_{1}^{3}+9 A_{3}^{3}\right)}{\left(1+\left(3 \varepsilon_{2} A_{1}^{2} / 2\right)-\omega^{2}\left(9+5 \varepsilon_{1} A_{1}^{2}\right)\right.} \\
& \omega^{2}=\frac{1+\left(3 \varepsilon_{2} / 4\right)\left(A_{1}^{2}+A_{1} A_{3}+2 A_{3}^{2}\right)}{1+\left(\varepsilon_{1} / 2\right)\left(A_{1}^{2}+3 A_{1} A_{3}+10 A_{3}^{2}\right)}
\end{aligned}
$$

Eqs. 25 and 26 are solved numerically for a given amplitude $A$, using an iterative technique with an accuracy of $10^{-6}$.

\section{RESULTS AND DISCUSSION}

The derived non-linear non-dimensional uni-modal equation of motion given in (20) is valid for vibrations with large amplitudes and small rotations, i.e. $\left(\lambda v^{\prime}\right)^{2}<<1$, which is the case in structures with high slender ratio.

The coefficients of the terms $\beta_{i}$ given in Eq. 19 are calculated by integrating numerically the coefficients given in Eqs. 14-17. Also, it is worth mentioning that the range of motion amplitudes to be considered in the present work, (i.e., the values of vibration amplitude $A$ ), is assumed to be up to 1.0 for the first mode, 0.4 for the second mode and 0.2 for the third mode, to be consistent with the assumption of large amplitude vibration. For example, a vibration amplitude of 1 corresponds to a ratio of tip displacement/length of the beam.

The accuracy of the calculated nonlinear natural frequencies was first examined by comparing the results obtained using: the Harmonic Balance method using single (SHB) and two terms (2THB) given in Eqs. 22 and 26, for the double tapered beam and $\alpha=b_{0} / b_{1}=0.1$, as shown in Figure 1. Results were obtained and presented in Figures 3-5, for the first three modes. As one, can see the SHB fails to predict the correct nonlinear natural frequency, specially for the second and third mode, and the 2THB method is more accurate. Consequently, all the remaining results were obtained using the method of Harmonic Balance method with two terms (2THB).

In Figures 6-8, results were obtained for the double tapered beam and for different values of the taper ratio $\alpha=b_{0} / b_{1}$. Results have shown that the behavior of the first and second modes is changed from hardening to softening when the taper ratio is increased, while the third mode is of a softening type regardless the value of the taper ratio $\alpha$. This is due to the fact that when the taper ratio $\alpha$ increases the mode shape is modified accordingly, which in turn affects the values of the calculated coefficients $\beta_{i}$ given in Eq. 19 and the values of $\varepsilon_{1}$ and $\varepsilon_{2}$. 
1st Mode, $\alpha=0.1$

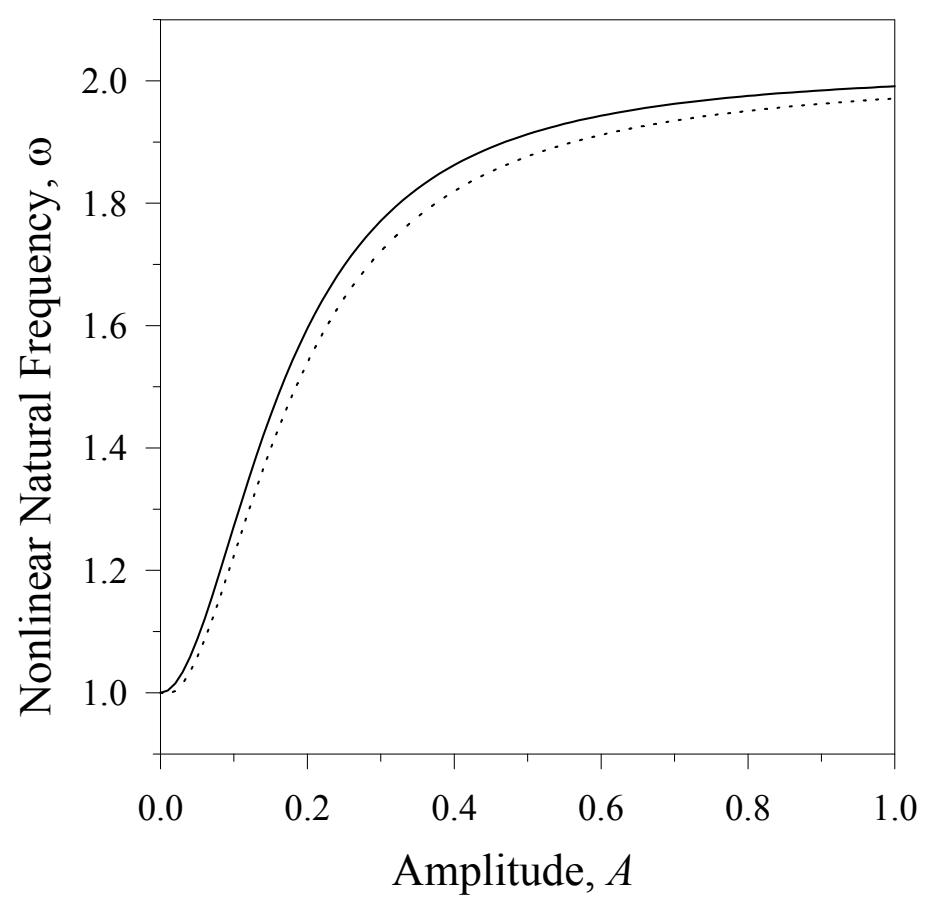

Figure 3. Nonlinear Natural Frequency Versus Amplitude of the First Mode, Double Tapered Beam and for $\alpha=0.1$

2nd Mode, $\alpha=0.1$

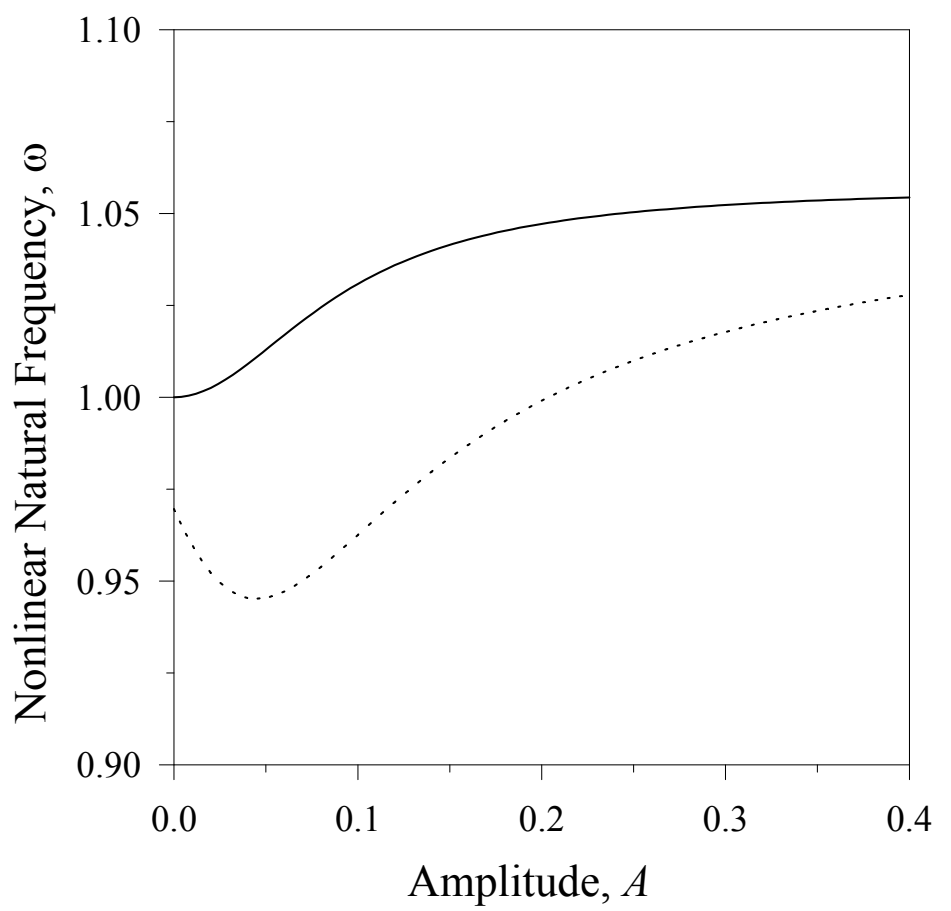

Figure 4. Same as in Figure 3, but for the Second Mode 
3rd Mode, $\alpha=0.1$

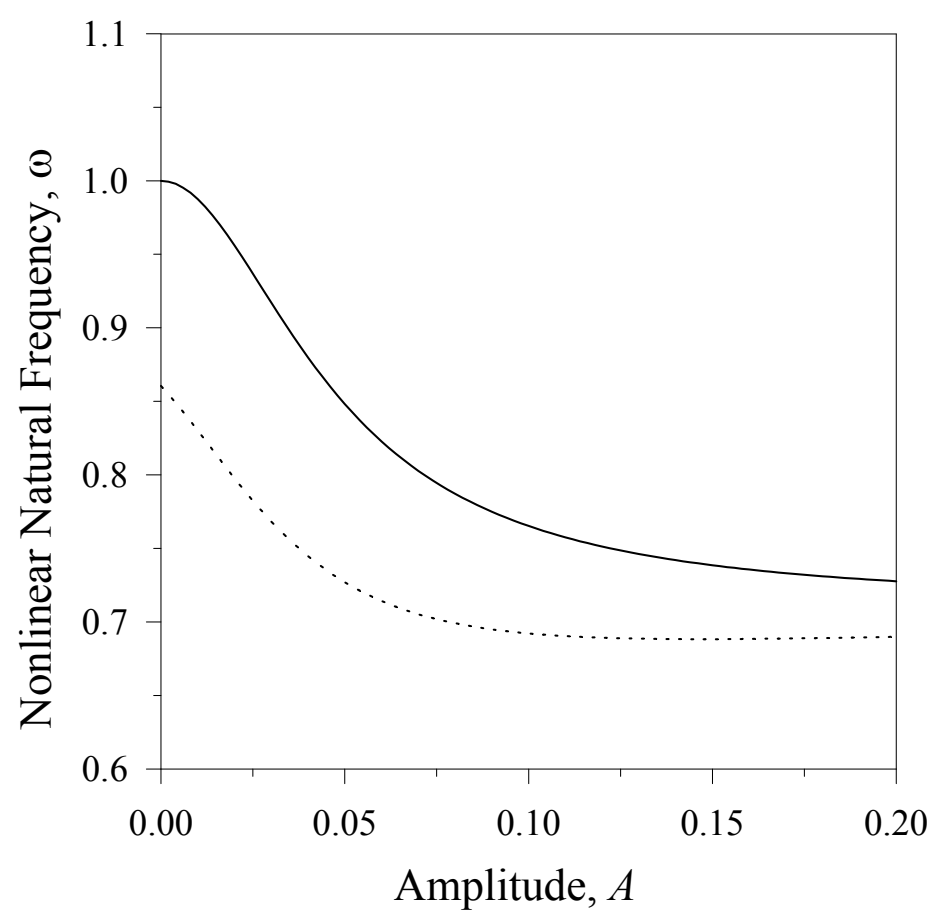

Figure 5. Same as in Figure 3, but for the Third Mode

1st Mode, $\alpha=0.1,0.2,0.3,0.4,0.5$

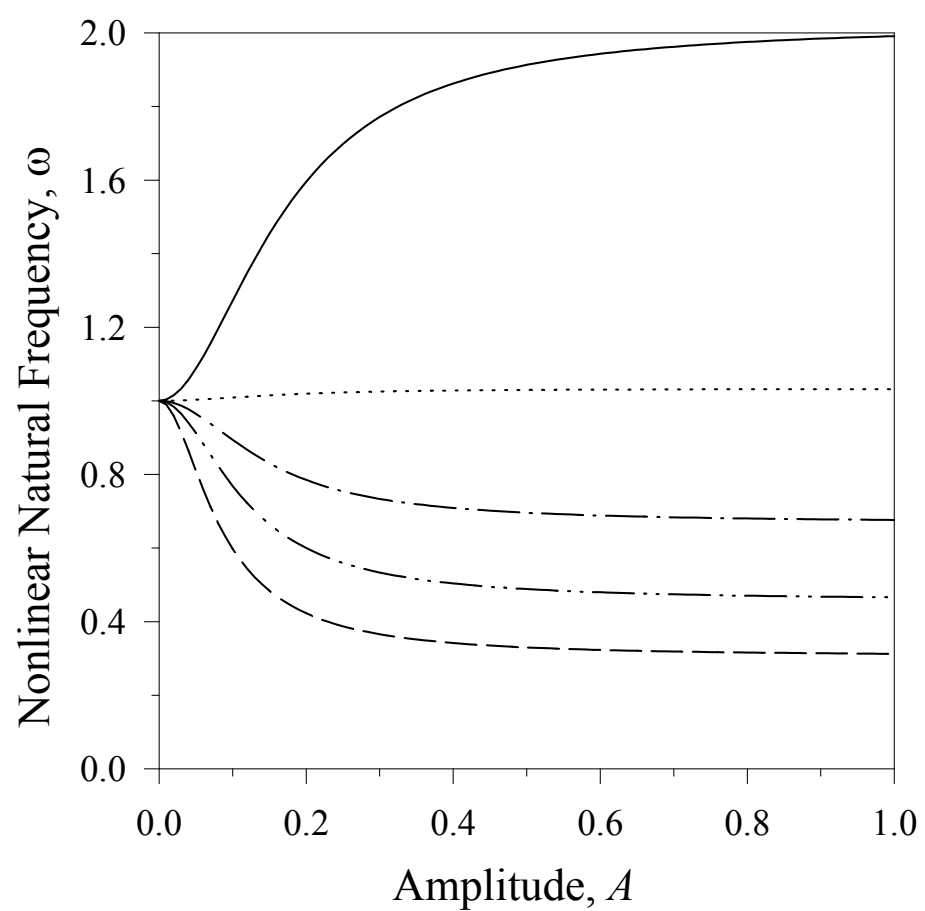

Figure 6. Nonlinear natural frequency versus Amplitude of the first mode, double tapered.

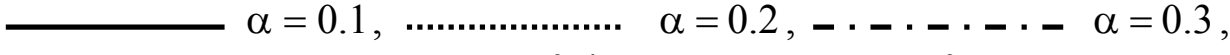


2nd Mode, $\alpha=0.1,0.2,0.3,0.4,0.5$

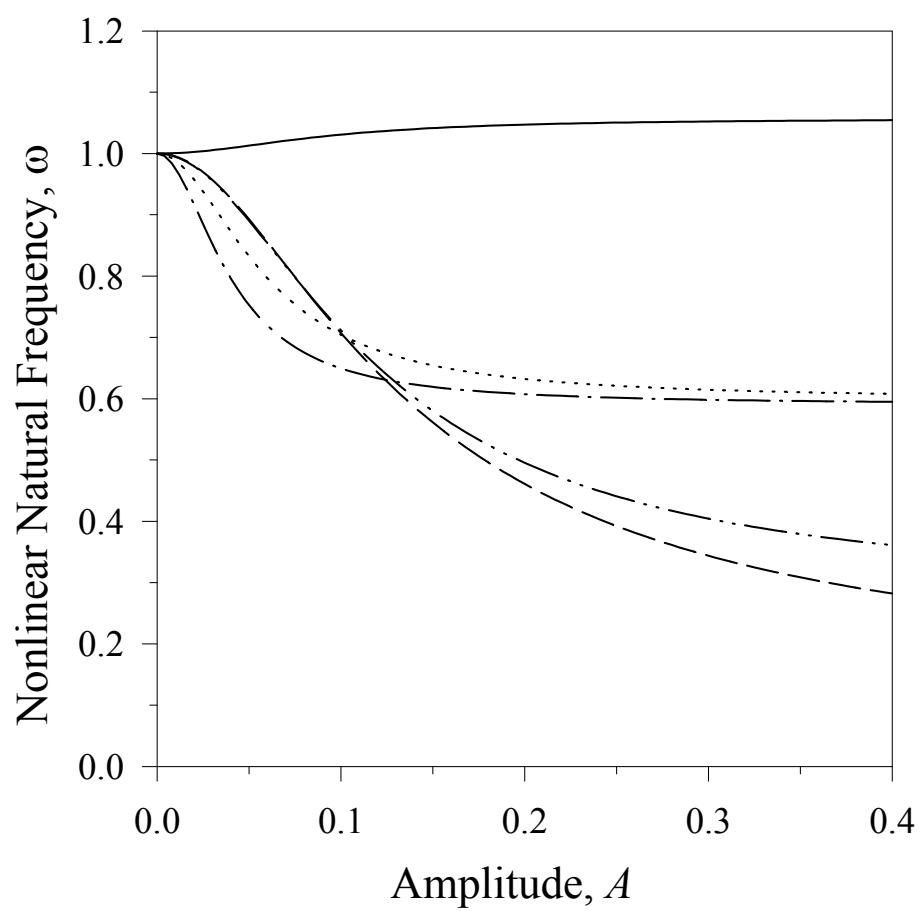

Figure 7. Same as in Figure 6, but for the second mode 3rd Mode, $\alpha=0.1,0.2,0.3,0.4,0.5$

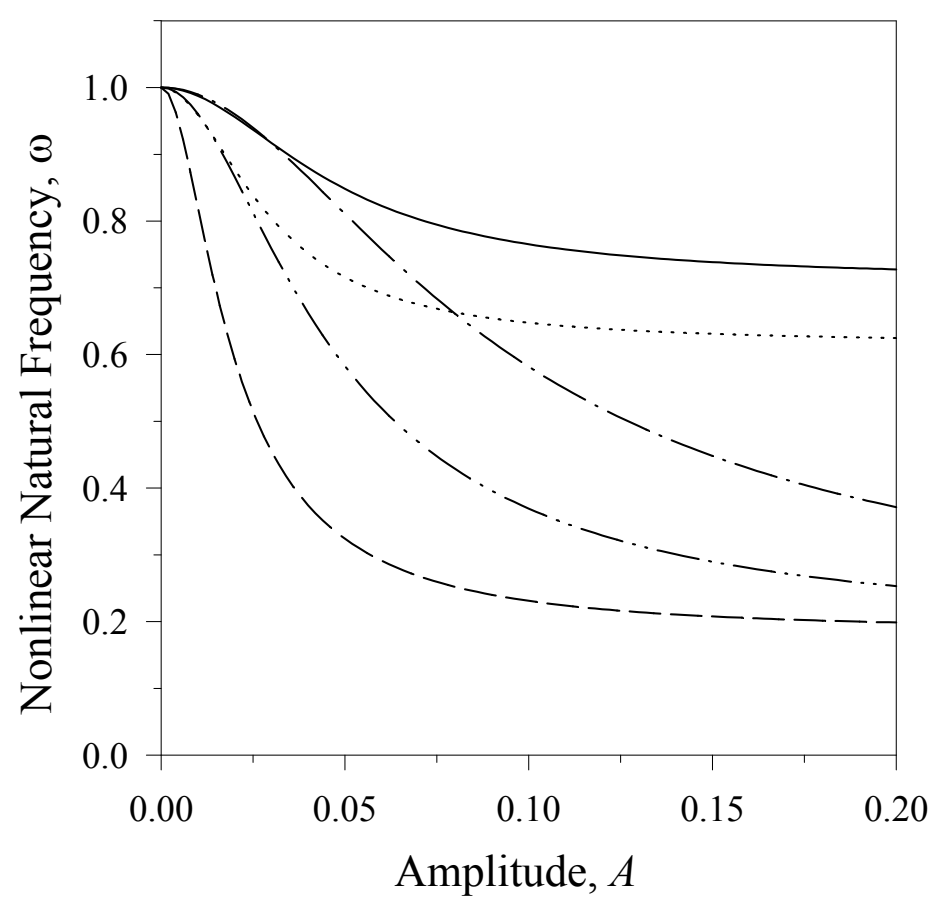

Figure 8. Same as in Figure 6, but for the third mode 
The nonlinear equation of motion given in Eq. 20, as mentioned before, is dominated by the non linearities; $\left(\varepsilon_{1} \ddot{q} q^{2}\right.$ and $\left.\varepsilon_{1} q \dot{q}^{2}\right)$ and $\varepsilon_{2} q^{3}$, inertia and static nonlinearities, respectively and the behavior is of hardening type when the ratio $\left(\varepsilon_{1} / \varepsilon_{2}\right) \leq 1.6$ and of softening type when $\left(\varepsilon_{1} / \varepsilon_{2}\right)>1.6[10]$.

In Figures 9-11 a comparison between the double tapered and wedge type beams for different values of taper ratio $\alpha=b_{0} / b_{1}$ is presented also. Results have shown that for a given value of taper ratio $\alpha$, the nonlinear natural frequency of a double tapered beam is higher than that of a wedge type "single taper beam".

1 st Mode, $\alpha=0.1,0.3,0.5$

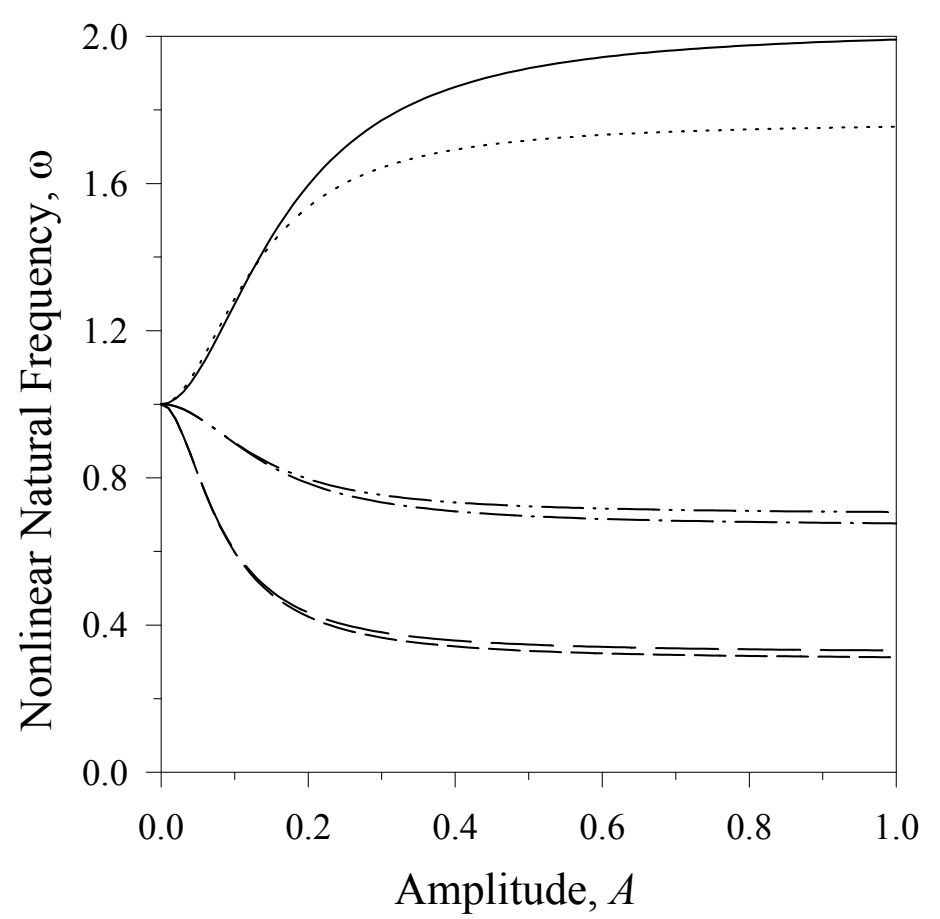

Figure 9. A comparison between the double tapered beam (D) and "wedge" single tapered (S) of the Nonlinear natural frequency versus Amplitude of the first mode.

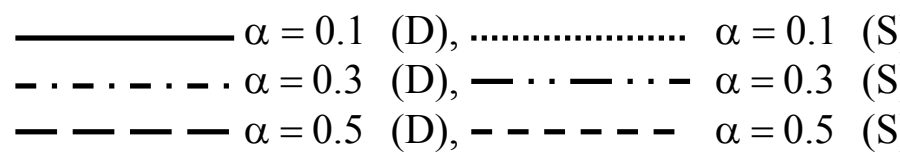




$$
\text { 2nd Mode, } \alpha=0.1,0.3,0.5
$$

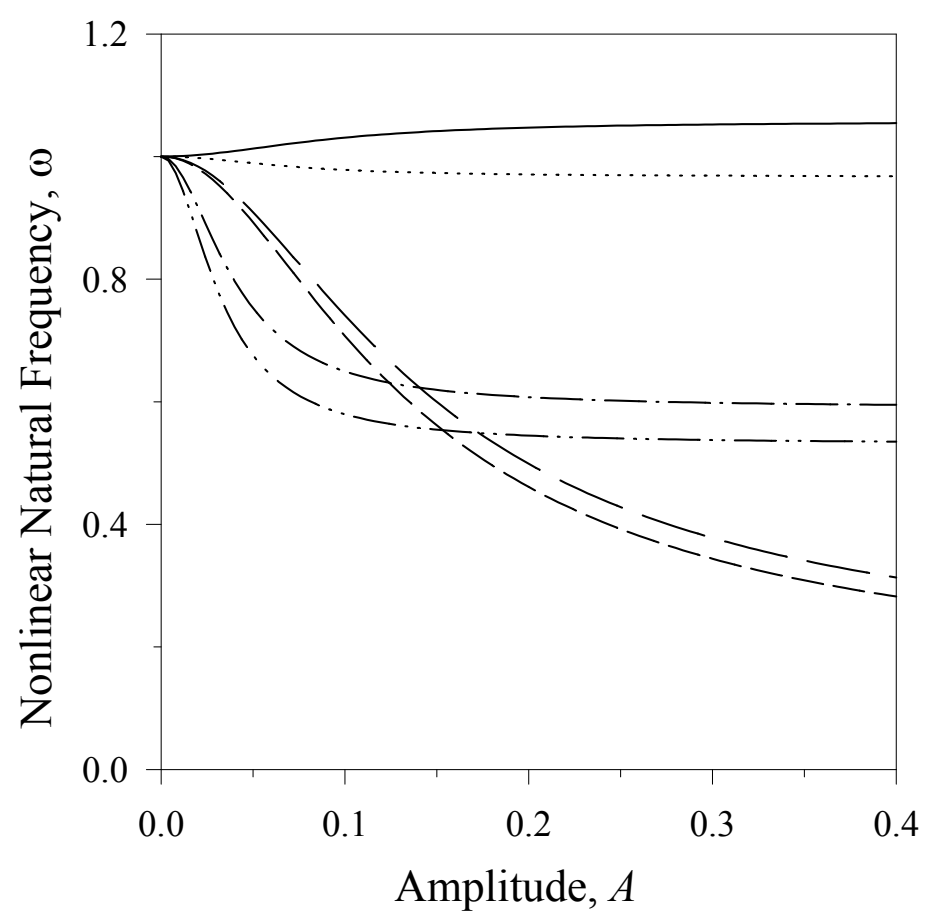

Figure 10. Same as in Figure 9, but for the second mode.

$$
3 r d \text { Mode, } \alpha=0.1,0.3,0.5
$$

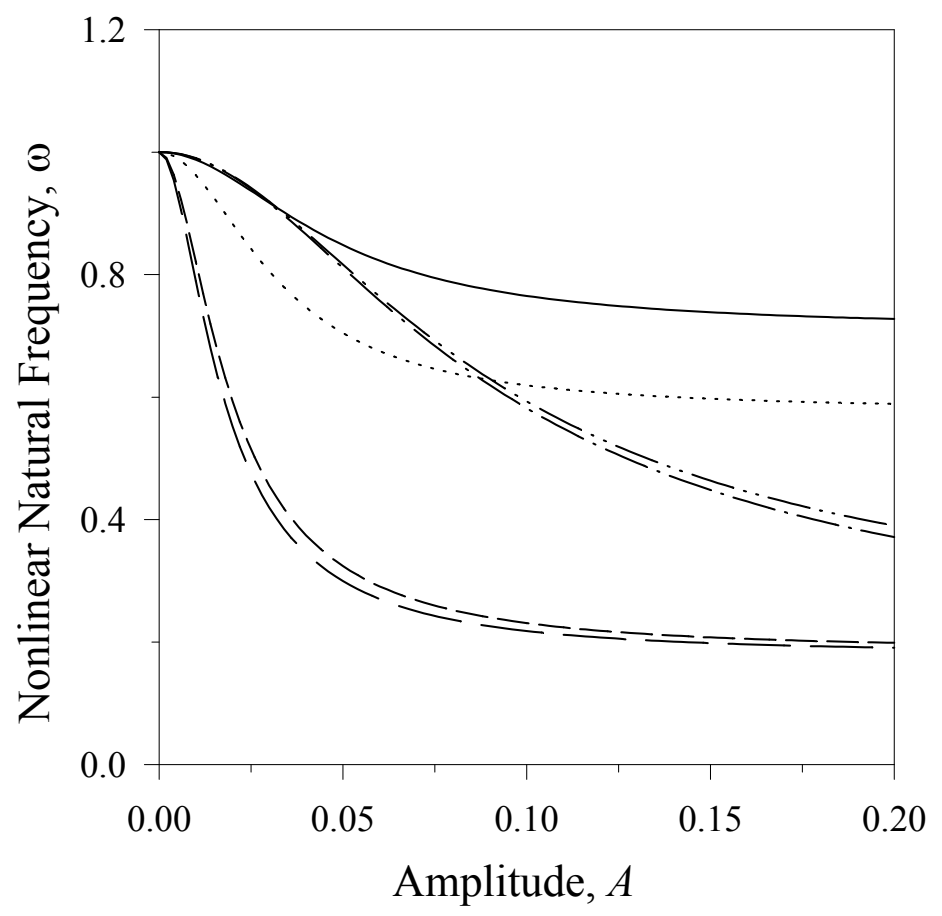

Figure 11. Same as in Figure 9, but for the third mode. 


\section{CONCLUSIONS}

A mathematical model for calculating the nonlinear natural frequencies of a tapered cantilever beam is derived. The axial shortening due to transverse deflection and the nonlinear curvature are used in the formulation of the kinetic and potential energy, respectively. The assumed mode method is used to discretize the continuous Lagrangian of the system and the resulted uni-modal nonlinear differential equation of motion is solved using the Harmonic Balance method (HB) to calculate the nonlinear natural frequencies for the first three modes of vibrations and for different values of the taper ratio $\alpha=b_{0} / b_{1}$ for two types of beams; double taper and single taper "wedge shaped".

Results have shown that for the first and second modes the behavior is changed from hardening to softening type when the taper ratio $\alpha$ is increased, while the third mode is of a softening type regardless the value of the taper ratio $\alpha$. Also, for a given value of a taper ratio, the nonlinear natural frequency of a double tapered beam is higher than that of a single tapered beam.

From the results presented for the effect of taper ratio $\alpha$ on the nonlinear natural frequencies, a qualitative change was noticed, i.e. when the nonlinear natural frequency changes from hardening type to a softening type. This would require a more detailed analysis to study the dynamic response of the beam under a given excitation load which is currently under consideration and beyond the scope of the present work.

\section{ACKNOWLEDGMENT}

Dr. Ahmad AL-Qaisia acknowledges the support of the Deanship of Academic research at the University of Jordan.

\section{REFERENCES}

[1] Auciello, N.M. and Nole, G., "Vibrations of a Cantilever Tapered Beam with Varying Section Properties and Carrying a Mass at the Free End", Journal of Sound and Vibration, 1998, Vol. 214, pp. 105-119.

[2] Nagaya, K. and Hai, Y., "Seismic Response of Underwater Members of Variable Cross Section", Journal of Sound and Vibration, 1985, Vol. 119, pp. 119-138.

[3] Laura, P.A. and Gutierrez, R.H., "Vibrations of an Elastically Restrained Cantilever Beam of Varying Cross Sections with Tip Mass of Finite Length", Journal of Sound and Vibration, 1986, Vol. 108, pp. 123-131.

[4] Shong, J.W. and Chen, C.T., "An Exact Solution for the Natural Frequency and Modes Shapes of An Immersed Elastically Wedge Beam Carrying an Eccentric Tip Mass with Mass Moment of Inertia", Journal of Sound and Vibration, 2005, Vol. 286, pp. 549-568.

[5] Chen, D.W. and Wu, J.S., "The Exact Solutions for the Natural Frequency and Modes Shapes of Non-Uniform Beams with Multiple Spring-Mass Systems", Journal of Sound and Vibration, 2002, Vol. 255, pp. 299-322.

[6] Goorman, D.J., "Free Vibrations of Beams and Shafts", John-Wiley \& Sons, 1975, pp. 365.

[7] Rao, B.N. and Rao, G.V., "Large Amplitude Vibrations of a Tapered Cantilever Beam", Journal of Sound and Vibration, 1988, Vol. 127, pp. 173-178. 
[8] Al-Qaisa, A.A., Shatnawi, A., Abdel-Jaber, M.S., Abdel-Jaber M. and Sadder, S., "Non-Linear Natural Frequencies of a Tapered Cantilever Beam",. Proceedings of the Sixth International Conference on Steel and Aluminum Structures (ICSAS'07), Oxford, UK, July 24-27, 2007, pp. 266-273.

[9] Al-Qaisia, A.A., "Effect of Fluid Mass on Non-Linear Natural Frequencies of a Rotating Beam", Proceedings of ASME Pressure Vessels and Piping Conference PVP2003, Cleveland, OHIO, USA, July 20-24, PVP-2003-2178, pp. 243-249.

[10] Al-Qaisia, A. A. and Hamdan, M. N., "On the Steady State Response of Oscillators with Static and Inertia Non-Linearities", Journal of Sound and Vibration, 1999, Vol. 223, pp. 49-71.

[11] Al-Qaisia, A.A. and Hamdan, M.N., "Bifurcation and Chaos of an Immersed Cantilever Beam in a Fluid and Carrying an Intermediate Mass", Journal of Sound and Vibration, 2002, Vol. 253, pp. 859-888. 